\title{
Selective medium for thermophilic campylobacters including Campylobacter upsaliensis
}

\author{
S T Aspinall, D R A Wareing, P G Hayward, D N Hutchinson
}

\begin{abstract}
Aims-To develop a selective supplement for the recovery of thermophilic Campylobacter spp, including Campylobacter upsaliensis from faeces, using campylobacter blood free selective agar base as the growth medium.

Methods-Minimum inhibitory concentrations (MICs) of cefoperazone and of teicoplanin were determined for 51 strains of $C$ upsaliensis, 159 strains of other thermophilic Campylobacter spp, and for 66 Enterobacteriaceae (cefoperazone only). From these results a medium using campylobacter blood free selective agar base incorporating cefoperazone (8 $\mathrm{mg} / \mathrm{l})$, amphotericin (10 $\mathrm{mg} / \mathrm{l})$, and teicoplanin (4 mg/1)-CAT mediumwas formulated and compared with the commercially available campylobacter blood free selective medium (modified CCDA) for isolation of Campylobacter spp from 7000 human faecal specimens. The two media were also compared for the recovery of $C$ upsaliensis from 45 spiked human faeces.
\end{abstract}

Results-Isolation rates of Campylobacter spp other than $C$ upsaliensis were similar for both media, but the CAT medium alone recovered four of the five strains of $C$ upsaliensis from the faecal samples examined. From the spiked faeces specimens, recovery of $\boldsymbol{C}$ upsaliensis was increased by between $35 \%$ and over 200-fold on the CAT medium compared with the modified CCDA.

Conclusions-CAT selective agar was a suitable alternative medium to modified CCDA for the growth of thermophilic Campylobacter spp, including $C$ upsaliensis from faeces.

( Clin Pathol 1993;46:829-831)

Public Health Laboratory, Royal Preston Hospital, PO Box 202, Preston, Lancs PR2 4HG $S$ T Aspinall D R A Wareing P G Hayward D N Hutchinson Correspondence to: ST Aspinall, Department of Microbiology, Royal Albert Edward Infirmary, Wigan Lane, Wigan WNi 2NN.

Accepted for publication 22 April 1993 bacteraemia, and opportunist infections in immunocompromised individuals, ${ }^{3-8}$ with studies suggesting that $3-13 \%$ of all campylobacter diarrhoeal disease is associated with
In 1983 a group of thermophilic, catalase group belonged to a new species and the name Campylobacter upsaliensis proposed. ${ }^{2}$ More recently, several reports have suggested that $C$ upsaliensis is a potential this organism..$^{910}$ Dogs could be one possible source of infection. ${ }^{1112}$

To elucidate the aetiology of such infections, suitable culture media are required to determine the prevalence of such organisms. Fewer than $20 \%$ of strains, however, have been reported to grow on the currently used campylobacter selective media and a filtration method using a non-selective medium is the technique advocated for its isolation. ${ }^{89}$

\section{Methods}

DETERMINATION OF MINIMUM INHIBITORY

CONCENTRATIONS (MIC)

Antibiotics

Cefoperazone (Unipath Ltd, Basingstoke, England) and teicoplanin (Merrell Dow Pharmaceuticals Ltd, Uxbridge, England) were supplied as a freeze dried supplement and a powder, respectively.

\section{Micro-organisms}

The following organisms were tested: $\mathbf{5 1}$ strains of $C$ upsaliensis, 140 strains of $C$ jejuni subspecies jejuni, and 19 strains of $C$ coli isolated from dog, cat, and human faeces using the membrane filtration culture technique, ${ }^{13} 14$ followed by incubation for 48 hours at $37^{\circ} \mathrm{C}$ in a microaerobic atmosphere.

Strains of Enterobacteriaceae $(\mathrm{n}=66)$ obtained from human faeces using MacConkey agar (Unipath Ltd) were also examined.

Isolates of Campylobacter spp were identified by accepted methods, ${ }^{15}$ while Enterobacteriaceae were identified using API $20 \mathrm{E}$ (API bioMerieux, Basingstoke, England).

\section{Method}

Doubling dilutions of cefoperazone from $2 \mathrm{mg} / 1$ to $128 \mathrm{mg} / 1$ and teicoplanin from $8 \mathrm{mg} / 1$ to $64 \mathrm{mg} / 1$ were prepared in campylobacter blood free selective agar base (Unipath Ltd) containing an additional $0.8 \%$ (w/v) bacteriological agar No 1 (Unipath Ltd), resulting in a final agar concentration of $2 \%$. Isolates for testing were cultured on Columbia blood agar containing $5 \%(\mathrm{v} / \mathrm{v})$ whole horse blood for 48 hours at $37^{\circ} \mathrm{C}$ in a microaerobic atmosphere. The bacteria were then suspended in $0.1 \%$ bacteriological peptone and adjusted to an optical density of $0 \cdot 2$ absorbance units at $465 \mathrm{~mm}$ using a BioRad 3550 microplate reader (BioRad Laboratories Ltd, Hemel Hempstead, England). This absorbance is equivalent to $10^{9}$ colony forming units $/ \mathrm{ml}$, as determined by the method of 
Miles and Misra. ${ }^{16}$ The suspensions of organisms were then diluted 1 in 1000 and inoculated on to the antibiotic containing media together with an equivalent antibiotic free medium, using a Denley multipoint inoculator (Denley Instruments Ltd, Billingshurst, England) with a 21 pin head. All plates were incubated at $37^{\circ} \mathrm{C}$ for 48 hours in a microaerobic atmosphere after which the MIC was defined as the lowest concentration of antibiotic at which growth was completely inhibited. The Campylobacter spp were tested against both cefoperazone and teicoplanin, while the Enterobacteriaceae were only tested against cefoperazone.

FORMULATION OF A NEW SELECTIVE MEDIUM Campylobacter blood free selective base was prepared using $8 \mathrm{mg} / 1$ of cefoperazone, 10 $\mathrm{mg} / \mathrm{l}$ of amphotericin (Bristol-Myers Squibb Pharmaceuticals Ltd, Hounslow, England), and $4 \mathrm{mg} / 1$ of teicoplanin (CAT medium). Strains of Campylobacter spp included: $C$ upsaliensis ( $\mathrm{n}=40), C$ jejuni subspecies jejuni $(\mathrm{n}=40), C$ coli $(\mathrm{n}=10), C$ lari $(\mathrm{n}=10), C$ cryaerophila $(\mathrm{n}=3)$ and $C$ hyointestinalis $(\mathrm{n}=$ 3) were inoculated on to both the CAT medium and an equivalent antibiotic free medium, spread for isolated colonies, and incubated at $37^{\circ} \mathrm{C}$ for 48 hours in a microaerobic atmosphere. After incubation all cultures were examined for typical growth and colonial morphology.

\section{COMPARISON OF CAT AND MODIFIED}

CCDA-PRESTON MEDIUM FOR ISOLATION OF CAMPYLOBACTER SPP FROM HUMAN FAECES

From human faeces

Modified CCDA-Preston medium (campylobacter blood free selective agar base, containing $32 \mathrm{mg} / \mathrm{l}$ of cefoperazone and $10 \mathrm{mg} / 1$ of amphotericin (Unipath Ltd)) was compared with CAT medium for the isolation of Campylobacter spp from 7000 human faeces submitted to Preston Public Health Laboratory for routine culture.

Using a 1 in 10 dilution of faeces $(0.5 \mathrm{~g}$ in $5 \mathrm{ml})$ in $0.1 \%(\mathrm{w} / \mathrm{v})$ bacteriological peptone, the media were inoculated using a sterile swab, spread for discrete colonies, and incubated at $37^{\circ} \mathrm{C}$ for 48 hours in a microaerobic atmosphere. Any suspect colonies were identified as described before. ${ }^{15}$

Growth of all isolates of Campylobacter spp (and for the first 1000 specimens, all isolates other than Campylobacter spp) were graded: scanty (growth only in the primary inoculum); moderate (growth in the primary and

Minimum inhibitory concentrations of $C$ upsaliensis, $C$ jejuni subspecies jejuni, $C$ coli and Enterobacteriaceae to cefoperazone

\begin{tabular}{|c|c|c|c|c|c|c|c|}
\hline & \multicolumn{7}{|c|}{ MIC of cefoperazone (mg/1) } \\
\hline & $\leqslant 2$ & 4 & 8 & 16 & 32 & 64 & $>128$ \\
\hline $\begin{array}{l}C \text { upsaliensis (51) } \\
C \text { jejuni sspp jejuni (140) } \\
C \text { coli (19) }\end{array}$ & 3 & 0 & 1 & 3 & 20 & 12 & $\begin{array}{r}12 \\
140 \\
19\end{array}$ \\
\hline Enterobacteriaceae (66) & 53 & 1 & 12 & & & & \\
\hline
\end{tabular}

secondary streaks); and heavy (growth in all streaks).

\section{From spiked human faeces}

Strains of $C$ upsaliensis ( $\mathrm{n}=45$ ) were inoculated into 45 different faecal samples at final concentrations of (a) $10^{5}-10^{6} \mathrm{cfu} / \mathrm{g}$ and (b) $10^{7}-10^{8} \mathrm{cfu} / \mathrm{g}$. Modified CCDA and CAT media were inoculated and incubated as above and recovery of the isolates recorded.

\section{Results}

MIC DETERMINATIONS

Teicoplanin

All Campylobacter spp had MICs to teicoplanin of $>64 \mathrm{mg} / \mathrm{l}$ on the campylobacter blood free selective base.

\section{Cefoperazone}

Most $C$ upsaliensis strains (47 out of 51 ) had MICs of $\geqslant 16 \mathrm{mg} / 1$ to cefoperazone (table), whilst all strains of $C$ jejuni and $C$ coli had MICs of $>128 \mathrm{mg} / \mathrm{l}$. All 66 strains of Enterobacteriaceae tested has MICs of $\leqslant 8 \mathrm{mg} / 1$.

\section{GROWTH OF CAMPYLOBACTER SPP ON CAT MEDIUM}

All isolates of Campylobacter spp tested yielded equivalent growth on both the CAT selective medium and the antibiotic free medium.

\section{COMPARISON OF CAT WITH MODIFIED CCDA}

\section{From human faeces}

Selectivity (1000 faeces) The same amount of growth of contaminants was found on both media in $91 \%$ of cultures; on CAT medium a small, moderate, or heavy increase in isolates other than Campylobacter spp was seen in $5 \cdot 5 \%, 2 \%$, and $0.5 \%$ of cultures, respectively. Conversely, $1 \%$ of cultures gave a moderate increase in contaminants on the modified CCDA compared with CAT medium.

Isolation of Campylobacter spp (7000 faeces) From the 7000 faecal specimens, 229 isolates of Campylobacter spp were recovered. These comprised 209 strains of $C$ jejuni subspecies jejuni, 13 strains of $C$ coli, two strains of $C$ lari and five strains of $C$ upsaliensis. Both CAT and modified CCDA media yielded similar growths of Campylobacter spp (other than $C$ upsaliensis) with the CAT medium recovering 223 of the 224 isolates and the modified CCDA recovering 221 . Four of the strains of C upsaliensis isolates, however, were only recovered on the CAT medium and would not grow on the modified CCDA even after subculture.

From spiked human faeces

From the faecal specimens spiked with $C$ upsaliensis at a concentration of $10^{7}-10^{8} \mathrm{cfu} / \mathrm{g}$, the organism was recovered using CAT medium on 42 occasions, while it was isolated from 31 samples using modified CCDA. At a concentration of $10^{5}-10^{6} \mathrm{cfu} / \mathrm{g}$ $C$ upsaliensis was isolated from CAT and modified CCDA on 37 and 11 occasions, respectively. 
Discussion

Campylobacter upsaliensis has been implicated as a potential human pathogen by several workers, ${ }^{3-8}$ but its isolation from faeces using selective media has posed considerable problems due to the wide range of antibiotic susceptibilities observed for these organisms. ${ }^{8}$ The recommended method for isolation of $C$ upsaliensis involves a membrane filtration culture technique but this not only incurs the additional cost of media and filters, and the need to leave the inoculated filter on the agar for 45 minutes before removal and incubation, but also results in a lack of recovery of the organism from faeces containing less than $10^{5} \mathrm{cfu} / \mathrm{g} .{ }^{10}$ Therefore, we formulated a new selective supplement to be added to the commercially available campylobacter blood free selective agar base containing charcoal, which would permit the isolation of thermophilic Campylobacter spp and improve the recovery of $C$ upsaliensis from faeces. The reduced concentration of cefoperazone $(8 \mathrm{mg} / 1$ rather than $32 \mathrm{mg} / \mathrm{l}$ ) inhibited most Enterobacteriaceae, but not faecal streptococci; therefore teicoplanin $(4 \mathrm{mg} / \mathrm{l})$ was included. Amphotericin was also included as an antifungal agent at a concentration of $10 \mathrm{mg} / \mathrm{l}$, as used in the modified CCDA. At these concentrations, the newly formulated medium (CAT) did not affect the growth or colonial morphology of any of the Campylobacter spp tested, yet allowed the growth of 47 of the 51 isolates of $C$ upsaliensis tested.

In 1990 Goossens et al reported that 48 of 91 isolates of $C$ upsaliensis examined had MICs to cefoperazone of $\leqslant 6.25 \mathrm{mg} / \mathrm{l}$ when tested in Müller-Hinton broth. We found, however, that when cultured on a charcoal based solid agar medium only four of 51 isolates of $C$ upsaliensis had MICs of $\leqslant 8 \mathrm{mg} / \mathrm{l}$. One of the most likely explanations for this is the ability of charcoal to bind antibiotics with the result that the antibiotic effect is reduced.

When CAT medium was compared with modified CCDA for the isolation of thermophilic campylobacters from 7000 human faeces, isolation rates of Campylobacter spp other than $C$ upsaliensis were similar. Five $(2 \cdot 2 \%)$ of the 229 Campylobacter spp isolated, however, were $C$ upsaliensis and of these, four were isolated only on the CAT medium. The isolation rate for $C$ upsaliensis in this study was similar to that reported before from this laboratory, ${ }^{17}$ but some workers have reported that up to $13 \%$ of campylobacter diarrhoeal disease is associated with this organism. ${ }^{10}$

To investigate the recovery of $C$ upsaliensis using CAT medium compared with modified CCDA, 45 randomly selected campylobacter culture negative faecal samples were spiked with different strains of the organism at concentrations between $10^{5}$ and $10^{6} \mathrm{cfu} / \mathrm{g}$ and also between $10^{7}$ and $10^{8} \mathrm{cfu} / \mathrm{g}$. At the lower concentration $82 \%$ (37) of the strains were recovered on the CAT medium; only $24 \%$ (11) were recovered on modified CCDA. This particular concentration was chosen as it is approaching the level below which the membrane filtration culture technique has been reported to lack recovery from faeces. ${ }^{10}$ At the higher concentration, 93\% (42) of the isolates were recovered on the CAT medium whereas $69 \%$ (31) of strains were isolated on modified CCDA.

The ability of the newly formulated CAT supplement to inhibit the growth of faecal contaminants, when compared with the modified CCDA supplement, was slightly inferior to the latter. Nevertheless, the small increase in growth of other organisms-mainly Enterobacteriaceae- did not create difficulty in the recognition and hence the isolation of campylobacters.

This study has shown that $C$ upsaliensis can be isolated from human faeces using the CAT supplement and that the latter is as efficient as the modified CCDA supplement for the isolation of other thermophilic Campylobacter spp. Moreover, the need to use the membrane filtration culture technique has been reduced. Further work is continuing to compare CAT medium with membrane filtration for the recovery of $C$ upsaliensis from $\operatorname{dog}$ and cat faeces.

1 Sandstedt K, Ursing J, Walder M. Thermotolerant Campylobacter with no or weak catalase activity isolated from dogs. Curr Microbiol 1983;8:209-13.

2 Sandstedt K, Ursing J. Description of Campylobacter upsaliensis sp. nov. previously known as the CNW upsaliensis sp. nov. previously known
group. Syst Appl Microbiol 1991;14:39-45.

3 Steele TW, Sangster N, Lanser JA. DNA relatedness and biochemical features of Campylobacter spp isolated in Central and South Australia. $\mathcal{F}$ Clin Microbiol 1985;22: 71-4.

4 Patton CM, Shaffer N, Edmonds P, et al. Human disease associated with "Campylobacter upsaliensis" (catalasenegative or weakly positive Campylobacter species) in the United States. F Clin Microbiol 1988;27:66-73.

5 Lastovica AJ, LeRoux E, Penner JL. "Campylobacter upsaliensis" isolated from blood cultures of pediatric patients. $\mathcal{F}$ Clin Microbiol 1989;27:657-9.

6 Taylor DE, Hiratsuka K, Mueller L. Isolation and characterisation of catalase-negative and catalase-weak strains of Campylobacter species, including "Campylobacter upsaliensis" from humans with gastroenteritis. f Clin Microbiol 1989;27:2042-5.

7 Chusid MJ, Wortmann DW, Dunne WM. "Campylobacter upsaliensis" sepsis in a boy with acquired hypo gammaglobulinemia. Diagnost Microbiol Infect Dis 1990;13:367-9.

8 Goossens H, Pot B, Vlaes L, et al. Characterisation and description of "Campylobacter upsaliensis" isolated from human faeces. 7 Clin Microbiol 1990;28:1039-46.

9 Bolton FJ, Hutchinson DN, Parker G. Isolation of Campylobacter: What are we missing? $f$ Clin Pathol 1987;40:702-3.

10 Goossens H, Vlaes L, DeBoeck M. Is "Campylobacter upsaliensis" an unrecognised cause of human diarrhoea? upsaliensis" an unrecogn
Lancet 1991;337:1486-7.

11 Goossens H, Vlaes L, Butzler JP. Campylobacter upsaliensis enteritis associated with canine infections. Lancet 1991;337:1486-7.

12 Figura N. Campylobacter spp. isolated from dog faeces. Lancet 1991;338:1403.

13 Bolton FJ, Hutchinson DN, Coates D. Blood-free selective medium for isolation of Campylobacter jejuni from faeces. F Clin Microbiol 1984;19:169-71.

14 Hutchinson DN, Bolton FJ. An improved blood-free selective medium for the isolation of Campylobacte jejuni from faecal specimens. $\mathcal{F}$ Clin Pathol 1984;37: jejuni 7

15 Bolton FJ, Wareing DRA, Skirrow MB, Hutchinson DN Identification and biotyping of Campylobacters. In: Identification and biotyping of Campylobacters. In: Board RG, Jones D, Skinner FA, eds. Identification
methods in applied environmental microbiology. Oxford: methods in applied environmental microbiology.

16 Miles AA, Misra SS. The estimation of the bactericidal power of the blood. F Hyg (Camb) 1938;38:732.

17 Bolton FJ, Hutchinson DN, Parker G. Reassessment of selective agars and filtration techniques for isolation of Campylobacter species from faeces. Eur $\mathcal{f}$ Clin Microbio Infect Dis 1988;2:155-60. 